Louise F. Carey', Giles M. Anderson', Sanjay Kumar*1

\title{
A Novel Attention Bias Modification Single Session Training Improves Eye Gaze Behaviour in Social Anxiety Disorder: A Pilot Study
}

\author{
'Department of Psychology, Oxford Brookes University, Oxford, UK \\ *email: skumarlabrookes.ac.uk
}

DOI: 10.2478/gp-2020-0009

Received: 21 February 2020; Accepted: 11 March 2020

\begin{abstract}
Background: Attention bias modification (ABM) can reduce anxiety and attentional bias towards threatening stimuli, but evidence of its usefulness as a potential intervention for socially anxious individuals has been mixed. Eye contact avoidance, a maladaptive attentional strategy in social anxiety disorder (SAD), has yet to be targeted by ABM research.

Aims: This study sought to establish whether a new ABM training paradigm could increase attentional deployment towards eyes and what effect this would have on social and gaze-related anxiety.

Method: Participants $(n=23)$ recruited through adverts calling for people who felt anxious in social situations completed either a novel ABM training task designed to induce attentional bias towards images of eyes over images of noses, or control training. Data on response times (RTs), accuracy of responses, gaze behaviour (using an eye tracker) and scores on clinical measures of social and gazerelated anxiety were collected before and after both training tasks.

Results: ABM training produced a greater number of initial saccades towards eye images than did the control task, indicating an induced shift in early attentional deployment. ABM training was also associated with a marginal increase in fixation durations on eye images. No effect was observed on RTs or social and gaze-related anxiety.

Conclusions: Our results indicate that ABM can alter the gaze behaviour of socially anxious individuals. They also highlight the importance of eye tracking to ABM research, because it was more sensitive than analyses of RTs to changes in early attentional deployment.
\end{abstract}

\section{Keywords}

Social anxiety, Cognitive bias, Eye fixation, Attentional bias, Saccade

\section{INTRODUCTION}

Cognitive biases play a role in the onset and maintenance of many psychological disorders, including social anxiety disorder (SAD; Mathews \& MacLeod, 2002; Woud \& Becker, 2014). According to Clark and Wells' cognitive model of SAD (1995), biased attentional and interpretive processes influence negative cognitions, which in turn lower mood and affect behaviour. Researchers such as MacLeod theorised that, through systematic training, such cognitive biases could be induced and altered and that this cognitive bias modification (CBM) would result in emotional and behavioural changes (MacLeod, Rutherford, Campbell, Ebsworthy \& Holker, 2002). Research into the efficacy of CBM for altering cognitive biases and reducing anxiety has shown promising results (e.g. MacLeod et al., 2002; Amir, Weber, Beard, Bomyea \& Taylor, 2008).
Several types of CBM have arisen to target different cognitive biases, including cognitive bias modification for interpretation (CBM-I), which focuses on the interpretation of ambiguous information, and attention bias modification (ABM), which focuses on attentional processes (Woud \& Becker, 2014).

Studies with non-anxious participants provide strong support for ABM's effectiveness at altering both attentional biases and anxiety levels. MacLeod et al. (2002) induced cognitive biases in non-anxious participants using a modified version of the dotprobe paradigm. In a dot-probe task, participants are shown a pair of stimuli (e.g. a pair of words or images), followed by a probe (e.g. a dot or a letter) that appears in the same location as one of the stimuli. Participants have to respond to the probe as quickly as possible (e.g. by pressing a button), and their response times (RTs) are measured. In the context of $\mathrm{ABM}$ 
research, dot-probe tasks can be used to induce an attentional bias by training participants to selectively respond to negatively, positively or neutrally valenced stimuli.

For example, in MacLeod's study, participants were shown pairs of words, one of which was neutral (e.g. 'Saddle') and one negative (e.g. 'Cancer'). Each pair of words was followed by the appearance of either one or two dots in the location of either the neutral or the negative word, with participants being asked to indicate as quickly as possible how many dots had appeared. In the attend-negative condition, the $\operatorname{dot}(\mathrm{s})$ consistently appeared in the location of the negative word, training participants to attend to negative stimuli over neutral stimuli, whereas in the attend-neutral condition, the $\operatorname{dot}(\mathrm{s})$ appeared in the location of the neutral word, training for a bias away from negative and towards neutral stimuli. Following the training, both conditions exhibited the expected attentional bias towards either neutral or negative word. The attend-neutral condition also displayed lower anxiety levels than the attend-negative condition during a stress-provoking task.

Later studies have found that $\mathrm{ABM}$ remains effective when delivered outside of laboratory conditions and that it can reduce anxiety experienced during real-world stressful situations (e.g. See, MacLeod \& Bridle, 2009). ABM research with patients with SAD, however, has produced more mixed results (e.g. Liu, $\mathrm{Li}$, Han \& Liu, 2017). In one study, socially anxious participants who completed ABM training displayed reductions in anxiety that were linked to increased activity in the prefrontal cortex and reduced activation of the bilateral amygdala, neuronal activity similar to that induced by accepted anxiolytic interventions (Taylor et al., 2014). However, a more recent review of 34 multisession ABM studies found that ABM threatavoidance training and control attentional training often led to similar reductions in anxiety and that these anxiolytic effects were not accompanied by consistent reductions in attentional bias towards threat (Mogg, Waters \& Bradley, 2017). Enock, Hofmann and McNally's (2014) double-blind randomised controlled trial investigating the potential of delivering ABM via smartphone similarly found a significant reduction in anxiety in both $\mathrm{ABM}$ and control training conditions, whilst cognitive bias remained unchanged in either condition.

ABM may yet have potential as a treatment for SAD (e.g. Shafiei \& Zare, 2019), but further research is needed to develop more effective $A B M$ interventions tailored to social anxiety (Pelissolo, Abou Kassm \& Delhay, 2019; Liu et al., 2019). One area that studies examining the effects of $\mathrm{ABM}$ on social anxiety could usefully focus on is sources of anxiety that are specific to SAD. The fear of direct eye contact is a well-documented feature of SAD, yet it has received little attention in the literature on ABM for social anxiety. Socially anxious individuals can perceive direct gaze as threatening (Schneier, Rodebaugh, Blanco, Lewin \& Liebowitz, 2011). Moreover, patients with SAD show differential patterns of eye contact when looking at faces compared to typically functioning individuals, making less eye contact during social interactions and fewer fixations on the eyes of faces in eye tracking studies (Schneier et al., 2011; Schulze, Renneberg \& Lobmaier, 2013; Langer, Lim, Fernandez \& Rodebaugh, 2017).

Langer and Rodebaugh (2013) found that socially anxious participants who were instructed to make less eye contact partway through a social interaction experienced more anxiety than those instructed to make more eye contact or to keep levels of eye contact constant. They concluded that avoidance of eye contact may be an ineffective anxiety-reduction strategy for socially anxious individuals and one which could usefully be targeted in therapeutic interventions.

Considering the role of eye contact avoidance in SAD, the present study uses a novel ABM paradigm to attempt to induce an attentional bias towards images of eyes in individuals who experience social anxiety. The study addresses the following research questions: 1) can avoidance of direct eye contact in individuals with self-reported social anxiety be modified using eye gaze focussed ABM? 2) What effect will this have on social and gaze-related anxiety?

\section{METHOD}

\section{Participants}

A sample of 24 participants was recruited through advertisements calling for people who felt anxious or nervous in social situations. Participants were volunteers who were not compensated for their time. All participants provided written informed consent. Participants were allocated randomly to either the control or the ABM training condition. Both conditions were informed that the experiment was investigating patterns of eye contact in socially anxious individuals to see if it might be possible to alter these patterns, but participants were naïve as to the mechanisms underlying ABM.

One participant in the control condition did not complete the experiment and was excluded from the analysis. The remaining 23 participants were aged between 16 and 56 years $(\mathrm{ABM}$ training $\mathrm{M}=38.8$; control $\mathrm{M}=30.7$ ). Participant characteristics are presented in Table 1. Independent-samples t-tests found no significant differences in age, social and gaze-related anxiety 
Table 1. Participant characteristics by condition .

\begin{tabular}{|c|c|c|c|}
\hline Characteristic & ABM Training & Control Training & p \\
\hline Age & & 30.7 & .683 \\
\hline M & 32.8 & 12.5 & \\
\hline SD & 12.4 & & \\
\hline $\begin{array}{c}\text { Liebowitz Social Anxiety Scale Score } \\
\text { (pre-assessment) }\end{array}$ & & 53.6 & \\
\hline M & 55.6 & 27.1 & \\
\hline SD & 30.4 & & \\
\hline $\begin{array}{c}\text { Gaze Anxiety Rating Scale Score } \\
\text { (pre-assessment) }\end{array}$ & & 32.4 & \\
\hline M & 30.8 & 15.9 & \\
\hline SD & 21.0 & & \\
\hline Hamilton Depression Rating Scale Score & & & \\
\hline M & 8.73 & 3.97 & \\
\hline
\end{tabular}

Participant characteristics by condition $(\mathrm{M}=$ mean, $\mathrm{SD}=$ standard deviation $)$

and depression scores at pre-training between participants allocated to the two conditions. Mean Liebowitz Social Anxiety Scale (LSAS) scores at pre-training in both conditions were 30 or above, a cutoff score for probable SAD that has robust sensitivity and specificity (Mennin et al., 2002). All but four participants (two in the control condition and two in the ABM condition) scored 30 or above on the LSAS, indicating marked levels of social anxiety.

\section{Design}

The experiment consisted of three phases, all three of which took place within a single session. In the pre-training phase, participants were interviewed using the Hamilton Rating Scale for Depression (HAM-D; Hedlund \& Vieweg, 1979) to assess the level of depression and then completed the LSAS(Heimberg et al., 1999) and the Gaze Anxiety Rating Scale (GARS; Schneier et al., 2011) online using Qualtrics (https://www.qualtrics.com/ $\mathrm{uk} /$ ). Participants then completed a 100-trial assessment dotprobe task, whilst their RTs and eye movements were recorded to measure their attentional responses to the experimental stimuli. In the training phase, participants completed either ABM training or a control dot-probe task of 500 trials. In the post-training phase, the assessment dot-probe task, LSAS and GARS were completed again to assess for changes in attentional bias, social and gaze-related anxiety. RT and eye tracking data were recorded during the pre- and post-training tasks but not during the training phase itself.

The assessment, ABM training and control dot-probe tasks were all designed using the SR Experiment Builder program (SR Research Ltd, 2011) and used the same stimuli and basic structure. The stimuli were 50 photographs of eyes and 50 photographs of noses, measuring 264 pixels $\times 100$ pixels (visual angles of $6.15^{\circ} \times 2.33^{\circ}$ ), cropped from photographs of neutral faces taken from the Umeå University Database of Facial Expressions (Samuelsson, Jarnvik, Henningsson, Andersson, \& Carlbring, 2012) and the NimStim set of facial expressions (Tottenham et al., 2009). For the purposes of the eye tracking portion of the experiment, rectangular regions of interest (ROIs) measuring 304 pixels $\times 140$ pixels (visual angles of $7.08^{\circ}$ $\times 3.26^{\circ}$ ) were defined around both eye and nose images. The regions of interest were larger than the images to account for the spatial accuracy of eye tracking ( \pm 0.5 of a visual degree) and thus to allow for the capture of all fixations and saccades towards the stimuli.

The stimuli were presented on a display PC with a 23.6区 colour LED LCD monitor (520 $\mathrm{mm} \times 290 \mathrm{~mm}$, BenQ XL2410T) with an aspect ratio of $16: 9$ and a resolution of 1920 pixels $\times 1080$ pixels. Participants viewed the stimuli in a well-lit room with blacked-out windows to avoid changes in luminance. In the assessment tasks, participants were asked to place their heads on a chin rest at a distance of $650 \mathrm{~mm}$ from the screen, with the height adjusted so that eye gaze was central to the display screen. Calibration was performed before the two assessment tasks, and a drift correction to the calibration was made before each individual trial.

In each trial, participants viewed a fixation cross in the centre of the screen for $1,000 \mathrm{~ms}$, followed by a 1,000 ms presentation of two photographs, one of a pair of eyes and one of a nose. One image was positioned 100px above, and one 100px below, the fixation cross. A probe, either one or two asterisks, then 
appeared in the location previously occupied by either the upper or the lower image. Participants were instructed to respond to the probe as quickly as possible by clicking the left mouse button if the probe was one asterisk and the right mouse button if it was two asterisks; a standard computer mouse was used, and the probe remained on screen until a response was received. The assessment, but not the training task, also featured 10 catch trials, included to ensure that participants attended to the images as well as to the probes. These trials featured a pair of eye or nose images rather than one image of each type. Participants were instructed to press the middle mouse button in these catch trials, regardless of probe identity.

During the assessment tasks, eye movements were recorded using an Eyelink 1000 system running at a spatial accuracy of $.25^{\circ}-.5^{\circ}$, a spatial resolution of $.01^{\circ}-.05^{\circ}$, and a temporal resolution of 1,000 Hz. The eye-tracking camera was linked to a separate host PC to the one displaying the stimuli and was synchronised with the display PC via an Ethernet cable. Eyelink software was used to control the camera and collect data.

There were equal numbers of the two probe types, probes appeared with equal frequency above and below the fixation cross, and the two stimulus types were equally distributed above and below the cross in all tasks. In the control training task, probes appeared with equal frequency behind images of eyes and noses. In the ABM training task, probes appeared behind images of eyes in all 500 trials to train for greater attentional deployment towards images of eyes. Figure 1 shows an illustration of the set-up and temporal order of the task, with examples of the stimuli used, and Table 2 summarises the dotprobe procedure.

\section{Data Analyses}

The dependent variables examined were participants' RTs to probes, the mean duration of participants' fixations on eye and nose ROIs, the number of participants' saccades that ended on eye and nose ROIs, the number of participants' first saccades that ended on eye and nose ROIs and participants' scores on the LSAS and GARS. The dependent variables were selected in order to investigate (1) whether ABM training would lead to faster deployment of attention towards eyes (as measured by RTs to probes appearing behind eye images), (2) whether ABM training would lead to changes in gaze behaviour toward eyes (as measured by analyses of fixations on and saccades ending on these ROIs) and (3) whether ABM training would lead to changes in social and gaze-related anxiety (as measured by participants' scores on the LSAS and GARS). In our selection of eye tracking variables, we were interested both in the direction

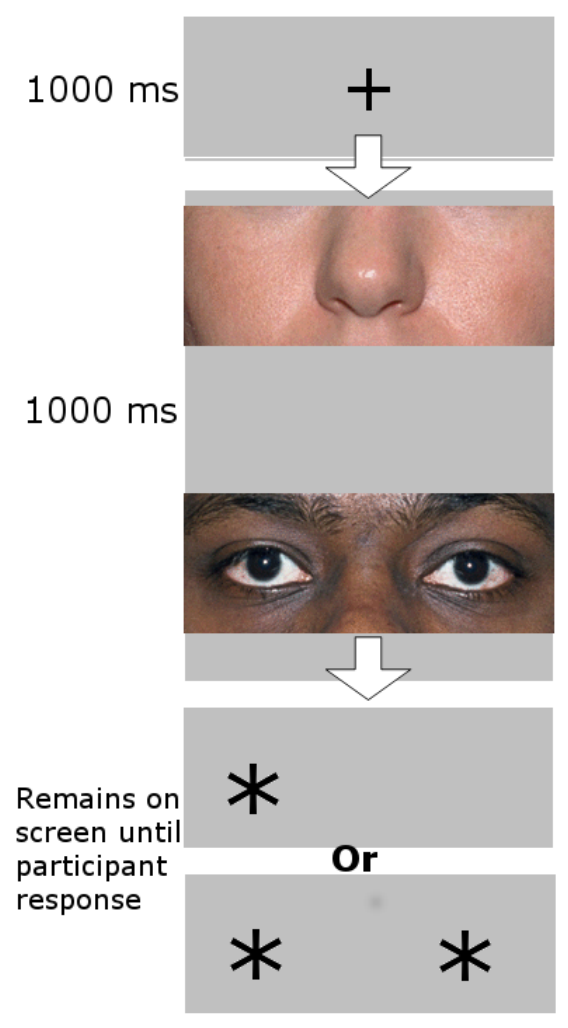

Figure 1. Illustration of experimental task set-up.

of gaze towards eyes (as measured by our analyses of saccades) and in the average length of participants' fixations on eyes (as measured by our analysis of mean fixation durations). These two eye tracking parameters allowed us to explore the initial orientation and sustenance of attention.

RTs, fixation durations and saccade frequencies were analysed using a series of three-way mixed factorial ANOVAs (condition $\times$ stimulus type $x$ time point). The within-subjects factors were time point (pre-training vs. post-training) and stimulus type (eyes vs. nose images), with condition (ABM vs. control) as the between-subjects factor. The time frames examined were from probe onset to participant response (as recorded by a mouse click) in the case of RTs, and the 1,000 ms from stimulus onset until probe onset in the case of fixations and saccades. In all cases, the result that is most relevant to the questions posed at the outset of this study is whether there is any three-way interaction between condition, stimulus type and time point. A significant interaction between these variables would indicate that ABM training and control training produced differential effects on participants' RTs or gaze behaviour, with these effects being moderated by stimulus type. 
Table 2. Table summarising the dot-probe task procedure in the pre-training, training and post-training phases

\begin{tabular}{|c|c|c|c|c|c|c|c|c|c|}
\hline Group & \multicolumn{3}{|c|}{ Pre-Training Assessment } & \multicolumn{3}{|c|}{ Training Phase } & \multicolumn{3}{|c|}{ Post-Training Assessment } \\
\hline Control & $\begin{array}{c}\text { Fixation } \\
\text { cross } \\
1000 \mathrm{~ms}\end{array}$ & $\begin{array}{l}\text { Eyes and nose } \\
\text { images above } \\
\text { and below } \\
\text { fixation cross. } \\
1000 \mathrm{~ms} \\
\text { Eyetracking } \\
\text { (incudes } 10 \\
\text { catch trials) }\end{array}$ & $\begin{array}{c}\text { One or two } \\
* \text { probe/s at } \\
\text { the location } \\
\text { previously } \\
\text { occupied by the } \\
\text { nose or eyes } \\
\text { image remain/s } \\
\text { on screen until } \\
\text { a response is } \\
\text { made. } \\
\text { RT and response } \\
\text { accuracy } \\
\text { collected. }\end{array}$ & $\begin{array}{c}\text { Fixation } \\
\text { cross } \\
1000 \mathrm{~ms}\end{array}$ & $\begin{array}{l}\text { Eyes and } \\
\text { nose } \\
\text { images } \\
\text { above and } \\
\text { below } \\
\text { fixation } \\
\text { cross. } \\
\text { 1000ms }\end{array}$ & $\begin{array}{c}\text { One or two } \\
\text { * probe/s at } \\
\text { the location } \\
\text { previously } \\
\text { occupied by the } \\
\text { nose or eyes } \\
\text { image remain/s } \\
\text { on screen until } \\
\text { a response is } \\
\text { made. }\end{array}$ & $\begin{array}{c}\text { Fixation } \\
\text { cross } \\
1000 \mathrm{~ms}\end{array}$ & $\begin{array}{l}\text { Eyes and nose } \\
\text { images above } \\
\text { and below } \\
\text { fixation cross. } \\
1000 \mathrm{~ms} \\
\text { Eyetracking } \\
\text { (incudes } 10 \\
\text { catch trials) }\end{array}$ & $\begin{array}{c}\text { One or two } \\
* \text { probe/s at } \\
\text { the location } \\
\text { previously } \\
\text { occupied by the } \\
\text { nose or eyes } \\
\text { image remain/s } \\
\text { on screen until } \\
\text { a response is } \\
\text { made. } \\
\text { RT and response } \\
\text { accuracy } \\
\text { collected }\end{array}$ \\
\hline $\begin{array}{c}\text { ABM } \\
\text { Training }\end{array}$ & $\begin{array}{c}\text { Fixation } \\
\text { cross } \\
1000 \mathrm{~ms}\end{array}$ & $\begin{array}{l}\text { Eyes and nose } \\
\text { images above } \\
\text { and below } \\
\text { fixation cross. } \\
1000 \mathrm{~ms} \\
\text { Eyetracking } \\
\text { (incudes } 10 \\
\text { catch trials) }\end{array}$ & $\begin{array}{c}\text { One or two } \\
* \text { probe/s at } \\
\text { the location } \\
\text { previously } \\
\text { occupied by the } \\
\text { nose or eyes } \\
\text { image remain/s } \\
\text { on screen until } \\
\text { a response is } \\
\text { made. } \\
\text { RT and response } \\
\text { accuracy } \\
\text { collected. }\end{array}$ & $\begin{array}{c}\text { Fixation } \\
\text { cross } \\
1000 \mathrm{~ms}\end{array}$ & $\begin{array}{l}\text { Eyes and } \\
\text { nose } \\
\text { images } \\
\text { above and } \\
\text { below } \\
\text { fixation } \\
\text { cross. } \\
\text { 1000ms }\end{array}$ & $\begin{array}{c}\text { One or two } \\
* \text { probe/s at } \\
\text { the location } \\
\text { previously } \\
\text { occupied by the } \\
\text { eyes image only } \\
\text { remain/s on } \\
\text { the screen until } \\
\text { a response is } \\
\text { made. }\end{array}$ & $\begin{array}{c}\text { Fixation } \\
\text { cross } \\
1000 \mathrm{~ms}\end{array}$ & $\begin{array}{l}\text { Eyes and nose } \\
\text { images above } \\
\text { and below } \\
\text { fixation cross. } \\
1000 \mathrm{~ms} \\
\text { Eyetracking } \\
\text { (incudes } 10 \\
\text { catch trials) }\end{array}$ & $\begin{array}{c}\text { One or two } \\
* \text { probe/s at } \\
\text { the location } \\
\text { previously } \\
\text { occupied by the } \\
\text { nose or eyes } \\
\text { image remain/s } \\
\text { on screen until } \\
\text { a response is } \\
\text { made. } \\
R T \text { and response } \\
\text { accuracy } \\
\text { collected }\end{array}$ \\
\hline
\end{tabular}

Italicised underlined indicates differences between the control and ABM training groups. .

LSAS and GARS scores were analysed with 2 two-way mixed factorial ANOVA (condition $\times$ time point). In this case, a two-way interaction between condition and time point would indicate that ABM training produced changes in participants' social and/or gaze-related anxiety.

Trials in which participants responded inaccurately to probes were excluded from all analyses. Trials in which participants' RTs were more than three standard deviations from the mean were excluded from the RT analysis but not the eye tracking analyses, because these responses were still accurate, indicating that attention had been paid to the stimuli. Three participants in the ABM condition did not produce any eye tracking data because of calibration problems and were excluded from the eye tracking analyses only. Fixations and saccades that began $80 \mathrm{~ms}$ or more before the onset of stimulus were also excluded from the eye tracking analyses as anticipatory (Anderson, Heinke \& Humphreys, 2013).

The accuracy rate of responses to probes was above $90 \%$ across condition, stimulus type and time point, and a threeway ANOVA on mean percentage accuracy rates found no significant main effects or interactions between any variables (all values for $p>.101$ ).

\section{RESULTS}

We report three sets of analyses: (1) mean RTs to probes, (2) mean duration of fixations on and frequency of saccades ending on eye and nose image ROIs and (3) LSAS and GARS scores at pre- and post-training.

\section{Response Times}

The mean RTs (ms) to eye and nose images for each condition are shown in Figure 2. A three-way ANOVA demonstrated the main effect of time point, $[F(1,21)=35.3, p<.001]$, with participants in both $\mathrm{ABM}$ and control conditions showing faster RTs to eye and nose images at post-assessment $(M=528)$ than pre-assessment $(\mathrm{M}=665)$. There was no main effect of condition, $[F(1,21)=.091, p=.766]$, or stimulus type, $[F(1,21)=$ $.104, p=.750]$. The two-way interactions (all values for $p>.190$ ) and the three-way interaction $[F(1,21)=.356, p=.557]$ were not significant.

\section{Eye Tracking Results}

Analyses were run on fixations on and saccades towards eye and nose ROIs. For the analyses of fixation durations, only 


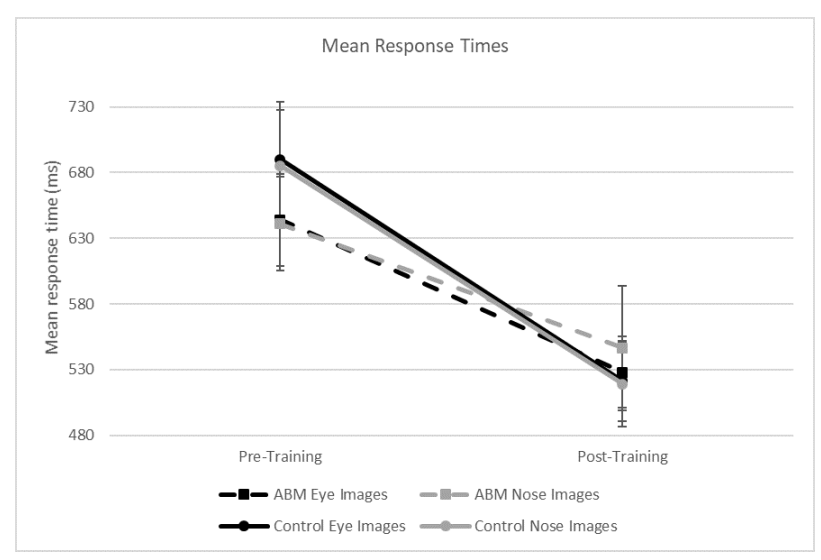

Figure 2. Mean response times (ms) to probes by stimulus type and time point in the ABM and control conditions lerror bars, in this and all other figures, indicate standard error]

times that fell within the window of stimulus presentation (i.e. the 1,000 ms from the onset of the images until the onset of the probe) were considered. When fixations continued past this window, their start time was subtracted from 1,000 to obtain their duration whilst the target stimulus was present, and these data were included in the analysis instead.

\section{Mean Fixation Durations}

The three-way ANOVA on mean fixation durations found no significant main effects of stimulus type, $[F(1,18)=1.67, p=.213]$, or condition, $[F(1,18)=.069, p=.769]$. There was a marginally significant three-way interaction (stimulus type $x$ time point $x$ condition, $[F(1,18)=3.08, p=.097])$. To understand this effect, we carried out planned comparisons for the ABM and control conditions separately and used Holm's sequential Bonferroni correction (Holm, 1979) for multiple comparisons, where the significance level of the first comparison remains at the same alpha level (.05 in our study) and subsequent alpha levels change based on the sequence of the comparisons (second comparison: $.05 / 2=.025$, third comparison: $.05 / 3=.0167$, and so on).

Fixation durations in the $\mathrm{ABM}$ condition increased significantly from pre- to post-training on eye $(p=.012)$ but not nose ( $p=$ .930) ROIs, whilst in the control condition, fixation durations did not increase following training towards either stimulus (noses: $p=.471$; eyes: $p=.682$ ). Furthermore, the ABM condition displayed significantly longer fixation durations on eye compared to nose ROIs at post-assessment ( $p=.040$ ), a difference not observed at pre-assessment $(p=.246)$ or in the control condition at either time point (pre-assessment: $p=.629$; post-assessment: $p=.699$ ). Mean fixation durations are shown in Figure 3.

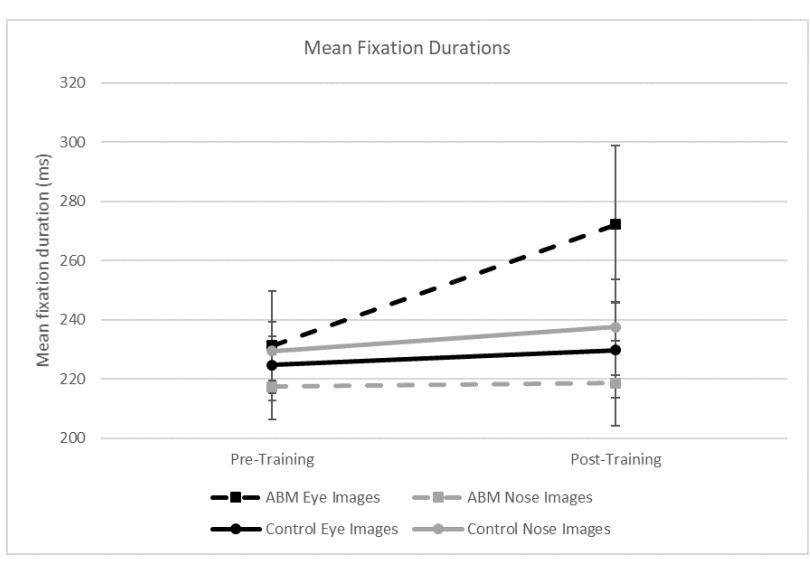

Figure 3. Mean fixation durations (ms) on ROls by stimulus type and time point in the $A B M$ and control conditions

The trend observed in the three-way interaction was also evident in a marginally significant two-way interaction between stimulus type and condition, $[\mathrm{F}(1,18)=3.52, p=.077]$. Participants in the ABM training condition displayed longer fixation durations on eye ROIs and shorter fixation durations on nose ROIs ( $\mathrm{M}=252$ vs. $\mathrm{M}=218, p=.012)$. The control condition did not show any such difference $(p>.05)$. There was also a marginally significant effect of time point, indicating an overall increase in mean fixation durations from pre-training $(\mathrm{M}=226)$ to post-training $(\mathrm{M}=239)$ across condition and stimulus type $[F(1,18)=4.24, p=.054]$. All other two-way interactions were non-significant (all values for $p>.152$ ).

\section{Saccade Frequency}

The number of saccades ending on eye and nose ROIs from pre- to post-training was examined in the two conditions. Saccades that were completed after the target stimuli had been replaced by the probe were excluded from the analysis $(1.85 \%$ of trials), because these saccades could not have ended with a fixation on either stimulus.

A three-way ANOVA demonstrated a main effect of time point, $[F(1,18)=5.37, p=.032]$, whereby saccade frequency decreased across condition and stimulus type from pre-training $(\mathrm{M}=$ 75.1) to post-training $(M=62.1)$. A main effect of stimulus type was also found, $[F(1,18)=17.751, p=.001]$, with more saccades ending on eye ROIs $(M=83.5)$ than on nose ROIs $(M=53.7)$. There was, in addition, a main effect of condition, $[F(1,18)=$ $4.70, p=.044]$, with participants in the ABM condition making more saccades to both ROIs $(\mathrm{M}=83.5)$ than participants in the control condition $(M=53.7)$. No higher-level interactions were significant (all values for $p>0.16$ ). 


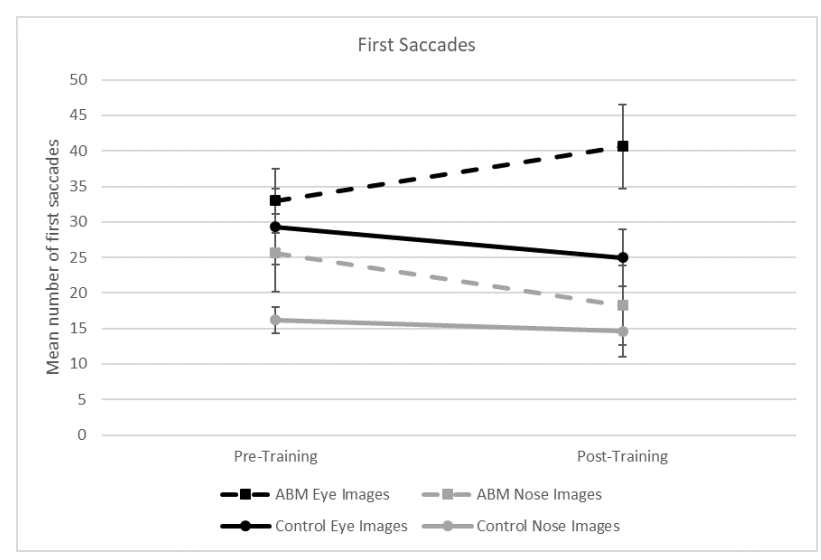

Figure 4. Mean number of first saccades ending on ROls by stimulus type and time point in the ABM and control conditions

\section{First Saccades}

To examine biases in participants' initial visual processing, it was decided to investigate the initial deployment of attention by analysing participants' first saccade in each trial. As such, a three-way ANOVA was run on first saccades that ended on eye and nose interest areas. Any saccades begun $80 \mathrm{~ms}$ or more before stimulus onset were excluded from this analysis as preparatory and replaced with the next earliest saccade beginning after $80 \mathrm{~ms}$. Participants mean first saccades by condition, time point and stimulus type are shown in Figure 4.

A significant three-way interaction was found amongst condition, stimulus type and time point, $[F(1,18)=6.36, p=$ .021]. The number of first saccades ending on eye and nose interest areas changed from pre-training to post-training, with this change being mediated by training condition. Planned comparisons (with a Bonferroni correction applied) revealed that the ABM condition made significantly more first saccades towards eyes than noses at post-training $(p=.003)$ but not at pre-training $(p=.171)$ and fewer first saccades towards noses at post-training than pre-training $(p=.037)$. The control condition made a greater number of first saccades towards eyes than noses at pre-training $(p=.006)$ but not at post-training $(p=.061)$. Furthermore, the ABM condition made more first saccades towards eyes at post-training than did the control condition ( $p=.035$ ). This three-way interaction demonstrates that $\mathrm{ABM}$ training (but not control training) led to an increase in initial saccades towards eye (but not nose) ROIs. This interaction is illustrated in Figure 4.

There was, in addition, a main effect of stimulus type, $[F(1,18)$ $=16.19, p=.001]$, whereby a greater number of first saccades were directed towards eyes $(M=32.0)$ than towards nose ROIs $(\mathrm{M}=18.7)$, but no effect of time point, $[F(1,18)=.500, p=.488]$, or condition, $[F(1,18)=2.744, p=.115]$. There was a marginally significant interaction between stimulus type and time point, $[F(1,18)=3.029, p=.099]$, whereby first saccades increased towards eyes (pre-training $M=31.2$ vs. post-training $M=32.8$ ) and decreased towards noses (pre-training $\mathrm{M}=20.9$ vs. posttraining $M=16.4$ ), from pre- to post-training across conditions. There were no other significant two-way interactions between any variables (all values for $p>.451$ ).

\section{LSAS and GARS Scores}

The ANOVA analysis on LSAS scores found no main effects of either time point, $[F(1,21)=.451, p=.509]$, or condition, $[F(1,21)$ $=.048, p=.829]$. There was also no interaction between the two, $[F(1,21)=.081, p=.778]$, demonstrating that neither ABM nor control training had any effect on LSAS scores.

The ANOVA analysis on GARS scores similarly found no main effects of condition, $[F(1,21)=.158, p=.695]$, or time point, $[F(1,21)=.084 p=.775]$, and no interaction between the two, $[F(1,21)=1.27, p=.273]$; neither ABM nor control training produced a change in GARS scores from pre- to post-training.

\section{DISCUSSION}

This study set out to establish whether (1) a novel eye gazebased ABM procedure could increase attentional deployment towards eyes in people with self-reported social anxiety and (2) what effect this would have on social and gaze-related anxiety.

Our results showed that first saccades towards eyes increased following ABM training but not control training, demonstrating that our ABM training paradigm successfully modified early attentional deployment towards eyes. Furthermore, the ABM training produced a marginally significant change in the expected direction in participants' mean fixation durations, with fixation durations on eye ROIs increasing following training in the ABM but not the control group. However, this shift in attentional deployment was not replicated in analyses of participants' RTs or saccade frequency, indicating that the change achieved was limited to the initial deployment of attention. Nor did these attentional changes lead to changes in social or gaze-related anxiety, as evidenced by the stability of LSAS and GARS scores from pre- to post-training.

In addition, the overall pattern of our results suggests that individuals with social anxiety show a higher saccadic frequency towards images of eyes than noses, suggesting the possibility 
of an abnormal regulation of saccadic behaviour towards eyes. Importantly, both training conditions exhibited fewer saccades to both stimulus types following training, suggesting that both training tasks produced an improvement in this potentially abnormal saccadic behaviour.

When interpreting the implications of these results, it should be noted that our training paradigm differed from those used in previous $A B M$ research in its direction of induced attentional change. SAD is characterised by attentional bias towards socially threatening stimuli (Matthews \& MacLeod, 2002). Previous $\mathrm{ABM}$ research with socially anxious populations has generally trained attentional deployment away from threatening or towards neutral or positive stimuli when attempting to reduce levels of social anxiety (e.g. Enock et al., 2014). We sought, instead, to increase attention towards a socially threatening stimulus (i.e. eyes; Schneier et al., 2011), predicting a reduction in anxiety secondary to reducing the maladaptive attentional strategy of gaze avoidance (Langer \& Rodebaugh, 2013).

Our findings concerning changes in saccadic frequency, first saccades and fixation durations fit interestingly with research into atypical patterns of facial processing in SAD. Individuals with SAD have been found to display fewer and shorter fixations on eyes compared to age- and sex-matched controls. They also use a 'hyperscanning' strategy when viewing photographs of faces, characterised by fewer and shorter fixations and longer scanpaths (Horley, Williams, Gonsalvez \& Gordon, 2003). The higher saccadic frequency towards eyes observed in our study further suggests that socially anxious individuals engage in maladaptive hyperscanning specifically for eye cues. Crucially, any training designed to increase focussed attention (whether ABM training or an unmodified dot-probe paradigm) may modulate this hyperscanning behaviour. Participants' greater number of first saccades towards eyes and increased fixation duration on eyes following $\mathrm{ABM}$ training further indicates that ABM training may have induced a slight additional shift away from patterns of visual processing associated with social anxiety. These results are encouraging, because they support the potential of this novel eye gaze-based ABM paradigm to address maladaptive patterns of gaze behaviour in populations with high levels of social anxiety.

Although attentional training of both types (control and ABM) led to a reduction in overall saccadic frequency, the lack of an overall change in participants' LSAS and GARS scores from pre- to post-training in either condition suggests that a larger number of training sessions may be required to produce a clinically meaningful effect. We delivered a single session of ABM training. By contrast, See et al. (2009) delivered 15 days of ABM training, finding evidence of an induced attentional bias at the end of this period but not in between individual training sessions. Given the marginal increase in mean fixation durations on eyes following ABM training that we found, it is possible that a significant increase might have been detected with greater statistical power.

Furthermore, participants in our study did not show differential gaze behaviour towards eye and nose images at pre-training, and this lack of any pre-existing attentional bias away from eyes may also partially account for the lack of effect on LSAS and GARS scores. In a large-scale investigation of the predictors of successful responses to ABM in individuals with the generalised subtype of social anxiety disorder (GSAD), the only cognitive factor that predicted the effect of ABM on LSAS scores was participants' baseline level of attentional bias (Amir, Taylor \& Donohue, 2011). In this study, participants who displayed an initial attentional bias towards socially threatening stimuli showed a significant reduction in anxiety following ABM training to shift their attention away from threatening cues. In contrast, those who did not display an initial attentional bias towards threat did not respond to the training, showing similar LSAS scores after training to a control condition. Given these findings, it seems that ABM is more effective in terms of clinical outcome measures for individuals who display a bias opposite to that induced by the training. As such, ABM training to reduce eye contact avoidance might be expected to lead to reductions on social and gaze-related anxiety measures only in participants with a pre-existing bias away from eyes.

As well as providing a point of departure for further research into the potential of ABM to reduce eye contact avoidance, the current study has highlighted the importance of a consideration of gaze behaviour to investigations of ABM. Eye tracking studies have an important role to play in ABM research, because they can provide insights into the time frame of attentional processing that examinations of response latencies cannot offer (Schofield, Johnson, Inhoff, \& Coles, 2012). In this study, for example, our analysis of first saccades identified an effect of $\mathrm{ABM}$ on the initial deployment of attention that the analysis of response latencies, the standard assessment measure for many ABM studies, did not identify. Eye tracking is crucial - not only for research into ABM for eye contact avoidance but also in ABM research more generally - to understanding and ultimately enhancing the mechanisms by which effective attentional bias interventions operate.

Overall, our findings are noteworthy because they show reliable changes in the gaze behaviour of socially anxious individuals after a single training session using our ABM paradigm. 
Our paradigm was able to induce a shift in initial attentional deployment, and both ABM and control training also produced some improvement in reducing a high saccadic frequency towards eye images associated with maladaptive hyperscanning behaviour. These findings provide useful insights into the potential of ABM to modify eye contact avoidance in socially anxious individuals, offering initial evidence that this novel ABM paradigm can induce a bias towards eyes.

\section{Limitations of the current study}

Although the current study provided insights into the effects of a novel ABM training paradigm, it had several limitations that should be taken into account when interpreting our findings. The main limitation of the study was its small sample size, which limited its statistical power. The participants were not formally diagnosed with SAD, and only their self-reported responses to the LSAS were used as a measure of their social anxiety. The study used a single session of ABM training when many of the most positive effects of $A B M$ training have been seen in multisession training protocols. Several factors contribute towards the effects of multisession training, and as such, our findings from a single session of training should be interpreted with caution. Finally, in the absence of a control group without social anxiety, it is difficult to determine whether or not the eye gaze behaviour observed in our study is specific to SAD.

\section{Directions for future research}

Future research in this area could focus on enhancing the effect of ABM training on attention towards eyes and investigating subsequent changes, if any, in social and gaze-related anxiety. It is possible that attentional bias is more successfully induced over a series of training sessions than within a single session, so future research might usefully compare different concentrations and time frames of ABM training to establish which produce the largest and most sustained changes in attentional bias. Furthermore, as social anxiety is usually observed in the context of anticipated or existing dynamic social interactions, a training protocol involving dynamic eye gaze behaviour change might be a useful avenue to explore.

\section{DECLARATIONS}

This research received no specific grant from any funding agency, commercial sectors or not-for-profit sectors. The study was approved by the Psychology Research Ethics Committee of Oxford Brookes University (reference number 1415/109 Carey).
The authors abided by the Ethical Principles of Psychologists and the Code of Conduct set out by the APA (http://www. apa.org/ethics/code/). All participants involved in this study provided written informed consent. The authors have no conflict of interest with respect to this publication.

\section{Authors' contributions}

LFC: conceptualisation of the study, experimental design and programming, data collection, data analysis, writing the manuscript; GMA: experimental design and programming, data analysis, feedback on the MS; SK: conceptualisation of the study, experimental design, data analysis, writing the manuscript, overall supervision.

\section{Acknowledgements}

The authors would like to thank Camden Ford, Rebecca Banks, Mike and Linda Carey, Wakefield Morys-Carter, and Dr Adam Lonsdale for their feedback and assistance.

\section{Conflict of interest}

The authors have no conflict of interest with respect to this publication.

\section{Ethical approval}

The study was approved by the Psychology Research Ethics Committee of Oxford Brookes University (reference number 1415/109 Carey). The authors abided by the Ethical Principles of Psychologists and the Code of Conduct set out by the APA (http://www.apa.org/ethics/code/).

\section{Funding}

This research received no specific grant from any funding agency, commercial or not-for-profit sectors.

\section{Informed consent}

All participants involved in this study provided written informed consent.

\section{Study registrations}

The study was not externally registered. 


\section{REFERENCES}

Amir, N., Taylor, C. T., \& Donohue, M. C. (2011). Predictors of response to an attention modification program in generalized social phobia. Journal of Consulting and Clinical psychology, 79(4), 533-541.

Amir, N., Weber, G., Beard, C., Bomyea, J., \& Taylor, C. T. (2008). The effect of a single-session attention modification program on response to a public-speaking challenge in socially anxious individuals. Journal of Abnormal psychology, 117(4), 860-868.

Anderson, G. M., Heinke, D., \& Humphreys, G. W. (2013). Topdown guidance of eye movements in conjunction search. Vision Research, 79, 36-46.

Clark, D., \& Wells, A. (1995). A cognitive model of social phobia. In Heimberg, R.; Liebowitz, M.; Hope, D.; Schneier, F. (Eds.), Social phobia: Diagnosis, Assessment, and Treatment (pp. 69-94). New York: The Guildford Press.

Enock, P. M., Hofmann, S. G., \& McNally, R. J. (2014). Attention bias modification training via smartphone to reduce social anxiety: A randomized, controlled multi-session experiment. Cognitive Therapy and Research, 38(2), 200-216.

Hedlund, J., \& Vieweg, B. (1979). The Hamilton rating scale for depression: a comprehensive review. Journal of Operational psychiatry, 10(2), 149-165.

Heimberg, R. G., Horner, K. J., Juster, H. R., Safren, S. A, Brown, E. J., Schneier, F. R., \& Liebowitz, M. R. (1999). Psychometric properties of the Liebowitz Social Anxiety Scale. psychological Medicine, 29(1), 199-212.

Holm, S. (1979). A simple sequentially rejective multiple test procedure. Scandinavian Journal of Statistics, 6(2), 65-70.

Horley, K., Williams, L. M., Gonsalvez, C., \& Gordon, E. (2003). Social phobics do not see eye to eye: A visual scanpath study of emotional expression processing. Journal of Anxiety Disorders, 17(1), 33-44.

Langer, J. K., Lim, M. H., Fernandez, K. C., \& Rodebaugh, T. L. (2017). Social Anxiety Disorder is Associated with Reduced Eye Contact During Conversation Primed for Conflict. Cognitive Therapy and Research, 41(2), 220-229.
Langer, J. K., \& Rodebaugh, T. L. (2013). Social anxiety and gaze avoidance: Averting gaze but not anxiety. Cognitive Therapy and Research, 37(6), 1110-1120.

Liu, H., Li, X., Han, B., \& Liu, X. (2017). Effects of cognitive bias modification on social anxiety: A meta-analysis. PLoS One, 12(4).

MacLeod, C., Rutherford, E., Campbell, L., Ebsworthy, G., \& Holker, L. (2002). Selective attention and emotional vulnerability: Assessing the causal basis of their association through the experimental manipulation of attentional bias. Journal of Abnormal psychology, 111(1), 107-123.

Mathews, A., \& MacLeod, C. (2002). Induced processing biases have causal effects on anxiety. Cognition \& Emotion, 16(3), 331-354.

Mennin, D. S., Fresco, D. M., Heimberg, R. G., Schneier, F. R., Davies, S. O., \& Liebowitz, M. R. (2002). Screening for social anxiety disorder in the clinical setting: Using the Liebowitz Social Anxiety Scale. Journal of Anxiety Disorders, 16(6), 661-673.

Mogg, K., Waters, A. M., \& Bradley, B. P. (2017). Attention Bias Modification (ABM): Review of Effects of Multisession ABM Training on Anxiety and Threat-Related Attention in HighAnxious Individuals. Clinical psychological Science, 26.04.17. doi: 10.1177/2167702617696359.

Nosofsky, R. M., \& Alfonso-Reese, L. A. (1999). Effects of similarity and practice on speeded classification response times and accuracies: further tests of an exemplar-retrieval model. Memory \& Cognition, 27(1), 78-93.

Pelissolo, A., Abou Kassm, S., \& Delhay, L. (2019). Therapeutic strategies for social anxiety disorder: where are we now? Expert Review of Neurotherapeutics, 19(12).

Pessôa, C. V. B. B., Perez, W. F., Endemann, P., Huziwara, E. M., \& Tomanari, G. Y. (2006). Eye fixations to figures in a four-choice situation with luminance balanced areas: Evaluating practice effects. Journal of Eye Movement Research, 2(5), 1-6.

Samuelsson, H., Jarnvik, K., Henningsson, H., Andersson, J., \& Carlbring, P. (2012). The Umeå University Database of Facial Expressions: a validation study. Journal of Medical Internet Research, 14(5). 
Schneier, F. R., Rodebaugh, T. L., Blanco, C., Lewin, H., \& Liebowitz, M. R. (2011). Fear and avoidance of eye contact in social anxiety disorder. Comprehensive psychiatry, 52(1), 81-87.

Schofield, C. A., Johnson, A. L., Inhoff, A. W., \& Coles, M. E. (2012). Social anxiety and difficulty disengaging threat: Evidence from eye-tracking. Cognition \& Emotion, 26(2), 300-311.

Schulze, L., Renneberg, B., \& Lobmaier, J. S. (2013). Gaze perception in social anxiety and social anxiety disorder. Frontiers in Human Neuroscience, 7(December), 872.

See, J., MacLeod, C., \& Bridle, R. (2009). The reduction of anxiety vulnerability through the modification of attentional bias: a realworld study using a home-based cognitive bias modification procedure. Journal of Abnormal psychology, 118(1), 65-75.

Shafiei, H., \& Zare, H. (2019). Effectiveness of attention bias modification by computerized attention training on reducing social anxiety of adolescents. Advances in Cognitive Sciences, 21(2).

SR Research Ltd. (2011). SR Research Experiment Builder 1.10.165 [Computer software]. Mississauga, Ontario, Canada: SR Research Ltd.

Taylor, C. T., Aupperle, R. L., Flagan, T., Simmons, A. N., Amir, N., Stein, M. B., \& Paulus, M. P. (2014). Neural correlates of a computerized attention modification program in anxious subjects. Social Cognitive and Affective Neuroscience.

Tottenham, N., Tanaka, J. W., Leon, A. C., McCarry, T., Nurse, M., Hare, T. A.,... Nelson, C. (2009). The NimStim set of facial expressions: Judgments from untrained research participants. Psychiatry Research, 168(3), 242-249.

Woud, M. L., \& Becker, E. S. (2014). Editorial for the special issue on cognitive bias modification techniques: An introduction to a time traveller's tale. Cognitive Therapy and Research, 38(2), 83-88. 